\title{
Diagnostic value and clinical significance of MRI and CT in detecting lymph node metastasis of early cervical cancer
}

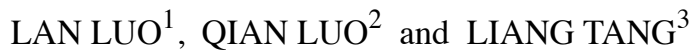 \\ Departments of ${ }^{1}$ Gynaecology, ${ }^{2}$ Radiology and ${ }^{3}$ Oncology, Jining No. 1 People's Hospital, \\ Jining, Shandong 272000, P.R. China
}

Received September 27, 2019; Accepted November 26, 2019

DOI: 10.3892/ol.2019.11180

\begin{abstract}
Diagnostic value and clinical significance of magnetic resonance imaging (MRI) and computed tomography (CT) in detecting lymph node metastasis of early cervical cancer were investigated. From April 2015 to April 2019, 160 patients with lymph node metastasis (stage I-II) of early cervical cancer in Jining No. 1 People's Hospital were retrospectively analyzed. All the patients underwent MRI, CT diagnosis and MRI combined with CT diagnosis before operation. Sensitivity, specificity and diagnostic accordance rate, the diagnostic value and clinical significance of MRI, CT and MRI combined with CT in the diagnosis of lymph node metastasis of early cervical cancer were compared. The sensitivity, specificity and diagnostic accordance rate of MRI in the diagnosis of lymph node metastasis of early cervical cancer in stage Ia-Ib were $75.00,72.92$ and $77.50 \%$, respectively, which were significantly higher than those of PET/CT in the same period $(\mathrm{P}<0.05)$. The sensitivity, specificity and diagnostic accordance rate of MRI combined with $\mathrm{CT}$ in the diagnosis of early cervical cancer in stage Ia-Ib were 78.13, 87.50 and $83.75 \%$, respectively, which were significantly higher than those of MRI or CT alone $(\mathrm{P}<0.05)$. However, the sensitivity, specificity and diagnostic accordance rate of MRI combined with $\mathrm{CT}$ in the diagnosis of lymph node metastasis of early cervical cancer in stage IIa-IIb were 91.66, 82.81 and 88.13\%, respectively, which were significantly higher than those of MRI or CT alone $(\mathrm{P}<0.05)$. MRI is superior to CT in the diagnosis of lymph node metastasis of early cervical cancer. However, the diagnostic efficiency of combined scans of the two is far higher than that of MRI or CT alone, which has more diagnostic value. In clinic, MRI and CT should be combined to improve the diagnostic accuracy of diseases.
\end{abstract}

Correspondence to: Dr Qian Luo, Department of Radiology, Jining No. 1 People's Hospital, 6 Jiankang Road, Jining, Shandong 272000, P.R. China

E-mail: eay4vx@163.com

Key words: MRI, CT, early cervical cancer, lymph node metastasis

\section{Introduction}

Cervical cancer is one of the main causes of cancer death in women (1), most of which is caused by human papillomavirus (HPV) infection (2). Approximately $86 \%$ of women who die from cervical cancer are in developing countries $(3,4)$. According to the clinical stage standard issued by FIGO, cervical cancer was staged based on the depth of tumor invasion and the degree of invasion to surrounding tissues (5). Patients with cervical cancer with FIGO stage I-II were divided into early cervical cancer (6). Although these were not mentioned in FIGO stage, lymph node metastasis has a very close relationship with the prognosis of cervical cancer and is the most important in cervical cancers (7).

Clinically, various imaging methods are used to detect lymph node metastasis in cancer, including magnetic resonance imaging (MRI) and computed tomography (CT) (8). $\mathrm{CT}$ imaging is very common in various clinical diagnoses. Its clinical value in tumor diagnosis and differential diagnosis cannot be ignored, and it has non-invasive advantages $(9,10)$. MRI has no radiation effect on human body. It can complete local and whole body scanning, which is more advanced and safe. Compared with CT, it also provides more anatomical details for diagnosis and the image contains more abundant texture information than CT images. Therefore, it is challenging to compare the advantages and disadvantages of MRI and CT. This needs to be analyzed on a case-by-case basis $(11,12)$. In this study, we compared the diagnostic value and clinical significance of MRI and CT in detecting lymph node metastasis of early cervical cancer.

\section{Patients and methods}

Collection of baseline data. In this study, we selected 160 patients with lymph node metastasis of cervical cancer from April 2015 to April 2019, including 122 patients with squamous cell carcinoma, 18 patients with adenosquamous carcinoma, 10 patients with endometrioid adenocarcinoma and 10 patients with clear cell carcinoma. The age of patients ranged from 28 to 64 years, with an average age of $35.59 \pm 4.02$ years. FIGO stage included 17 cases in stage Ia, 47 cases in stage Ib, 43 cases in stage IIa and 53 cases in stage IIb.

Inclusion criteria: i) The patient was diagnosed as cervical cancer by pathology after treatment in Jining No. 1 People's 
Hospital (Jining, China); ii) FIGO stage was stage I and II in patients. iii) Patients underwent plain scan to increase examination before operation (the scanning range included at least inferior diaphragm to pubic symphysis). iv) The patient had no other treatment before surgery. v) Patients selected radical hysterectomy bilateral salpingo-oophorectomy and pelvic lymphadenectomy, radical hysterectomy pelvic lymphadenectomy para-aortic lymphadenectomy. vi) Patients and their families signed informed consent forms in advance. The study was approved by the Ethics Committee of Jining No. 1 Hospital.

Exclusion criteria: i) Patients who received only plain scan or only pelvic plain scan and enhanced scan before operation. ii) The stage of FIGO was stage III or above and the postoperative pathology was cervical intraepithelial neoplasia. iii) Scan did not conform to the standard, patient examination preparation was not perfect or contrast agent injection factors did not conform to the diagnostic criteria. iv) The treatment method was radiotherapy, chemotherapy or other treatment methods. v) The patient received other preoperative treatment.

\section{Examination methods}

i) Main reagents and instruments: Siemens Verio 3.0T superconducting magnetic resonance was from Siemens. Gd-DTPA, a bolus contrast agent, ${ }^{18} \mathrm{~F}$-deoxyglucose $\left({ }^{18} \mathrm{FDG}\right)$, a CT imaging agent, were from Accdon. CT scanners were from Royal Philips Electronics. Sixty-four slice CT was from Siemens.

ii) CT examination methods: The patient should avoid, or consume only liquid food with little residue the day before the examination to ensure the formation of unformed feces. The enema was cleaned with warm water before examination for 1-2 h. Patients drank 600-800 $\mathrm{ml}$ warm boiled water to fill the bladder 30-60 min before examination to increase the contrast with the uterus. Retention enema was performed 5 min before CT scan: In order to fully dilate the rectum, the patient was placed in the left lateral position, taking the tolerance dose as the degree, and injected $\sim 400 \mathrm{ml}$ warm water through the anus with the intestinal irrigator bag to increase the contrast between the large intestine and the uterus. Training the patient's breathing before examination: It is required to hold the breath after inhaling calmly during examination, and to breathe calmly if the breath cannot be held. Before the examination, the technical operator explained to the patient the matters needing attention and checked whether the patient had any foreign matters affecting diagnosis, such as metal. The most commonly used volume of continuous scanning method was adopted: The tube voltage was $120 \mathrm{kV}$, the tube current was $300 \mathrm{~mA}$, the slice thickness was $5 \mathrm{~mm}$, the interlayer spacing was $5 \mathrm{~mm}$ and the reconstruction thickness was $1 \mathrm{~mm}$. The patient was placed in a supine position with both hands on the top of the head. The scanning range included at least inferior diaphragm to pubic symphysis. After conventional axial plain CT scan, the body position remained unchanged. Professional nurses injected $100 \mathrm{ml}$ of nonionic contrast agent through superficial vein of the elbow with high pressure injector at an injection rate of 2.5-3.0 ml/sec. Arterial phased scan was performed $\sim 30 \mathrm{sec}$ later, mainly scanning the liver. Portal phase enhanced scan was performed $\sim 65-75 \mathrm{sec}$, including whole abdomen or chest and abdomen and pelvic. Equilibrium phase can be added to scan when it was necessary according to lesion display and diagnostic needs.

iii) MRI examination methods: The examinee did not eat for $48 \mathrm{~h}$, and drank a proper amount of water to fill the bladder before 9 a.m. on the examination day. Then scan of $4.0 \mathrm{~mm} /$ layer was performed. The examination sequences were as follows: sagittal SET1WI, TR/TE 364/15 msec; sagittal TSE T2WI, TR/TE 2890/108 msec; sagittal fat suppression T2W(ISTIR), TR/TE3840/103 msec, TI $115 \mathrm{msec}$; oblique transverse T2WI of scanning diameter line and perpendicular to uterine axis, TR/TE4890/84 msec; Gd-DTPA T1WI transverse, TR/TE 397/15 msec; The sagittal scan parameters of T1WI were the same as those of plain scan.

The CT and MRI images were read by two radiologists with more than 10 years experience and no knowledge of the surgical and pathological results. The CT staging and MRI staging of cervical cancer were carried out according to the reading results.

Judgement of criterion. i) Image analysis of MRI diagnostic stage (Table I). ii) Image analysis of CT diagnosis of SC stage (Table II).

Statistical methods. Data analysis was completed by SPSS 18.0. The counting data were expressed as $[\mathrm{n}(\%)]$. The $\chi^{2}$ test was used for univariate analysis and comparison of the diagnostic accuracy of SC at each stage. The difference was statistically significant at $\mathrm{P}<0.05$.

\section{Results}

Baseline data. The basic conditions of 160 patients were investigated, such as age, obesity, smoking and drinking habits, histological classification, clinical stage (Fig. 1 and Table III). Degree of differentiation and lymph node metastasis of cervical cancer are shown in (Fig. 2).

\section{Diagnostic efficiency of MRI and CT in lymph node metas- tasis of early cervical cancer in different stages}

i) Diagnostic efficacy of MRI and CT in lymph node metastasis of early cervical cancer in stage I. The sensitivity, specificity and diagnostic accordance rate of MRI in the diagnosis of early cervical cancer in stage I were $75.00 \%, 72.92$ and $77.50 \%$, respectively. The sensitivity, specificity and diagnostic accordance rate of CT in the diagnosis of early cervical cancer in stage I were 62.50 , 56.25 and $58.75 \%$, respectively. Comparing data of the two groups, the sensitivity, specificity and diagnostic accordance rates of MRI in the diagnosis of early cervical cancer in stage I were significantly higher than those of CT $(\mathrm{P}<0.05)$ (Tables IV-VI).

ii) Diagnostic efficacy of MRI and CT in lymphatic metastasis of early cervical cancer in stage II. The sensitivity, specificity and diagnostic accordance rate of MRI in the diagnosis of lymphatic metastasis of early cervical cancer in stage II were $79.17,78.13$ and $78.75 \%$, respectively. The sensitivity, specificity and diagnostic accordance rate of 
Table I. Features of MRI of early cervical cancer in different stages.

\begin{tabular}{ll}
\hline Grouping & \multicolumn{1}{c}{ Features } \\
\hline Stage Ia & No evidence of mass lesion. \\
Stage Ib & Tumor pierced cervical low signal intensity ring. \\
Stage IIa & The tumor pierced the upper vagina but did not touch the parametrium. \\
Stage IIb & The matrix of the cervix was destroyed and the parametrium tissues were invaded. \\
\hline
\end{tabular}

MRI, magnetic resonance imaging.

Table II. Features of CT of early cervical cancer in different stages.

\begin{tabular}{ll}
\hline Grouping & \multicolumn{1}{c}{ Features } \\
\hline Stage Ia & No metastatic lymph nodes. \\
Stage Ib & Metastatic lymph nodes appeared, but were not obvious. \\
Stage IIa & Metastatic lymph nodes appeared but did not invade the parametrium. \\
Stage IIb & Metastatic lymph nodes began to invade the parametrium. \\
\hline
\end{tabular}

CT, computed tomography.

CT in the diagnosis of lymphatic metastasis of early cervical cancer in stage II were $62.50,68.75$ and $60.00 \%$, respectively. Comparing the data of the two groups, the sensitivity, specificity and diagnostic accordance rate of MRI in the diagnosis of lymphatic metastasis of early cervical cancer in stage II were significantly higher than those of CT in the diagnosis of lymphatic metastasis of early cervical cancer in stage II $(\mathrm{P}<0.05)$. However, there was no obvious difference in specificity $(\mathrm{P}>0.05)$ (Tables VII-IX).

Comparison of diagnostic efficiency between MRI combined with CT and MRI or CT alone in lymphatic metastasis of early cervical cancer in different stage. The sensitivity, specificity and diagnostic accordance rate of MRI combined with CT in the diagnosis of early cervical cancer in stage I were $78.13,87.50$ and $83.75 \%$, respectively, which were significantly higher than the sensitivity and diagnostic accordance rate of MRI or CT alone $(\mathrm{P}<0.05)$. However, the sensitivity, specificity and diagnostic accordance rate of MRI combined with $\mathrm{CT}$ in the diagnosis of early cervical cancer in stage II were 91.66, 82.81 and $88.13 \%$, respectively. There was no obvious difference in specificity $(\mathrm{P}>0.05)$, the specificity and diagnostic accordance rate were significantly higher than that of MRI or CT alone $(\mathrm{P}<0.05)$ (Tables X-XII).

\section{Discussion}

Cervical cancer is particularly harmful to women in developing countries, and screening its level is very important $(13,14)$. However, two imaging techniques, CT and MRI, play a vital role in the early stage of cancer, treatment strategy and treatment of response evaluation (15-17). In many studies on lung cancer, prostate cancer and breast cancer, MRI and CT are widely used (18-20). These technologies have their own advantages and disadvantages, and are suitable for examining different tumors (21). Therefore, the purpose of this study was to compare the diagnostic value of the two imaging methods in the detection of lymph node metastasis of early cervical cancer.

In this study, different stages of patients with early cervical cancer were examined with MRI, and CT alone, and MRI combined with CT, and their diagnostic efficacy such as sensitivity, specificity, diagnostic accordance rate was compared. According to the results, the sensitivity, specificity and diagnostic accordance rate of MRI in the diagnosis of lymph node metastasis of early cervical cancer with stage I (Ia-Ib) were significantly higher than those of CT in the diagnosis of lymph node metastasis of early cervical cancer with stage I ( Ia-Ib). In addition to specificity, the sensitivity, diagnostic accordance rate of MRI in the diagnosis of lymph node metastasis of early cervical cancer with stage II (IIa-IIb) were significantly higher than the sensitivity, specificity, and diagnostic accordance rate by $\mathrm{CT}$ diagnosis. In another study on cervical cancer, it was found that MRI is better than CT in diagnosing cervical cancer. It can not only be useful for the discovery of early cervical cancer, but also for reasonable staging (22). However, in the study of Li and Room (23) on invasive cervical cancer, MRI was shown to be better than CT in observing tumor size and boundary, the signal was easy to identify, and the diagnostic accordance rate for lymphatic metastasis was also very high. It was concluded that MRI has better diagnostic efficiency and higher clinical diagnostic value than CT in the diagnosis of lymph node metastasis of early cervical cancer.

In a study on lymphoma, it was found that MRI combined with CT has good examination effect (24). Therefore, in this 
Table III. Baseline clinical data of 160 SC patients.

\begin{tabular}{|c|c|}
\hline Factors & {$[\mathrm{n}(\%)]$} \\
\hline \multicolumn{2}{|l|}{ Age (years) } \\
\hline$\leq 50$ & $105(65.63)$ \\
\hline$>50$ & $55(34.37)$ \\
\hline \multicolumn{2}{|l|}{ Smoking } \\
\hline Yes & $81(50.63)$ \\
\hline No & $79(49.67)$ \\
\hline \multicolumn{2}{|l|}{ Drinking } \\
\hline Yes & $78(48.75)$ \\
\hline No & $82(51.25)$ \\
\hline \multicolumn{2}{|l|}{ Obesity } \\
\hline Yes & $80(50.00)$ \\
\hline No & $80(50.00)$ \\
\hline \multicolumn{2}{|c|}{ Histological classification of cervical cancer } \\
\hline Squamous cell carcinoma & $122(76.25)$ \\
\hline Adenosquamous carcinoma & $18(11.25)$ \\
\hline Endometrioid adenocarcinoma & $10(6.25)$ \\
\hline Clear cell carcinoma & $10(6.25)$ \\
\hline \multicolumn{2}{|l|}{ Clinical stage of cervical cancer } \\
\hline Stage Ia & $17(10.63)$ \\
\hline Stage Ib & $47(29.37)$ \\
\hline Stage IIa & $43(26.87)$ \\
\hline Stage IIb & $53(33.13)$ \\
\hline \multicolumn{2}{|l|}{ Differentiation degree } \\
\hline Poorly differentiated & $65(40.63)$ \\
\hline Middle differentiated & $45(28.12)$ \\
\hline Well differentiated & $50(31.25)$ \\
\hline \multicolumn{2}{|l|}{ Lymph node metastasis } \\
\hline Yes & $96(60.00)$ \\
\hline No & $64(40.00)$ \\
\hline
\end{tabular}

study, we also carried out combined examination of MRI and CT on patients. According to the results, besides the specificity index for diagnosing lymph node metastasis of early cervical cancer in stage II (IIa-IIb), the sensitivity and diagnostic accordance rate of MRI combined with CT diagnosis were significantly higher than those of MRI and CT alone. CT has good density resolution, can avoid intestinal peristalsis and other effects to a certain extent. It is visual and has certain advantages in finding lymph node diffusion, but its diagnostic sensitivity to cancer staging is not strong, and its differentiation specificity for lesions is not high. However, MRI has higher tissue resolution and better tissue contrast. It can directly display the tumor condition and can change imaging parameters to improve its contrast, thus improving the prediction range of tumors (25). Complementary advantages of the two can have a better examination effect. Therefore, we conclude that the diagnostic value of MRI and CT combined diagnosis was higher than that each alone. In the study of Kim et al (26) on non-small cell lung cancer, the combination of MRI and

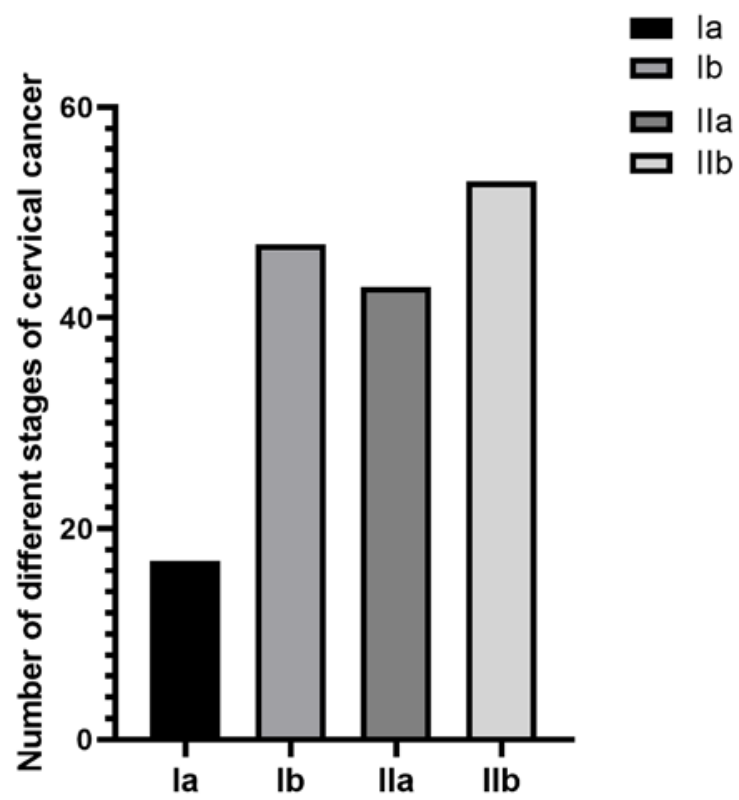

Figure 1. Number of early cervical cancer patients tested with four different stages.

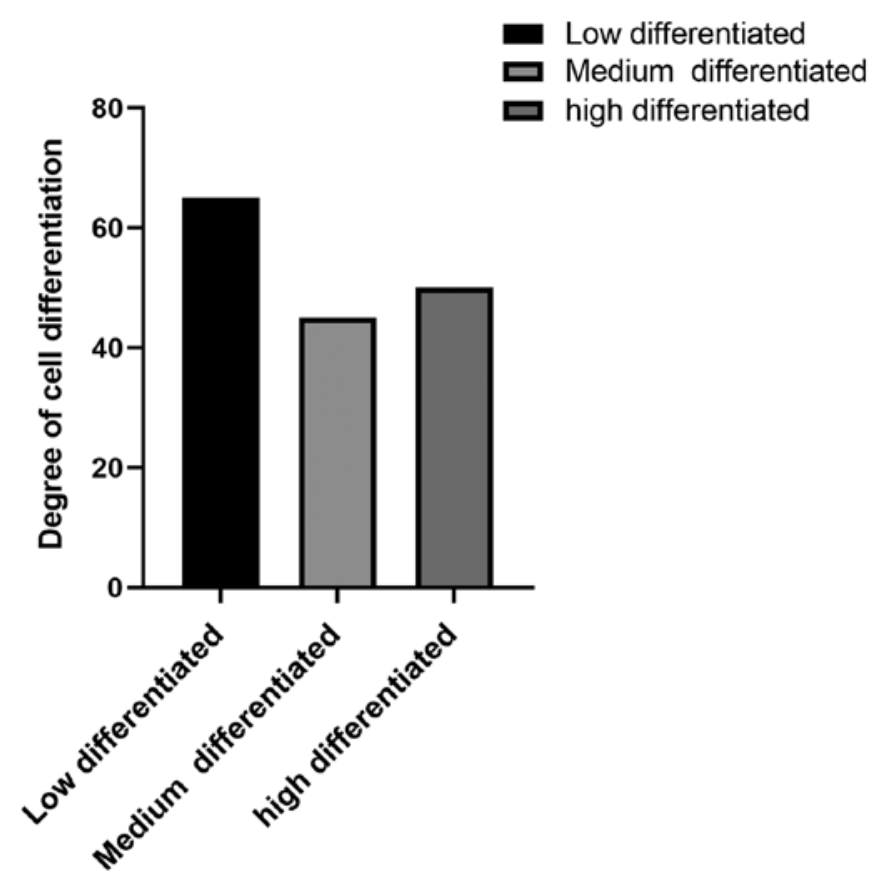

Figure 2. Differentiation of tumors.

CT was more helpful to significantly improve the sensitivity of detecting lymph node metastasis than $\mathrm{CT}$ alone. In a clinical study of calcified meningioma, it was found that CT combined with MRI diagnosis has a higher diagnostic accordance rate for tumors and a more comprehensive and obvious reflection on the characteristics of tumors (27). These are all similar to the results of the present study.

In conclusion, the diagnostic value of MRI in the clinical diagnosis of lymph node metastasis of early cervical cancer is significantly higher than that of CT and the sensitivity, specificity and diagnostic accordance rate are significantly 
Table IV. Diagnostic efficacy of MRI in lymph node metastasis of early cervical cancer in stage I.

Pathological diagnostic results

Grouping

Stage Ia-Ib

Stage non-Ia-Ib

Total

Ia-Ib phase by MRI diagnosis

54

26

80

Non-Ia-Ib phase by MRI diagnosis

10

70

Total

64

96

MRI, magnetic resonance imaging.

Table V. Diagnostic efficacy of CT lymph node metastasis of early cervical cancer in stage I.

Pathological diagnostic results

Grouping

Stage Ia-Ib

Stage non-Ia-Ib

Total

Ia-Ib phase by CT diagnosis

40

Non-Ia-Ib phase by CT diagnosis

24

54

Total

64

96

160

CT, computed tomography.

Table VI. Comparison of diagnostic efficiency of MRI and CT in lymphatic metastasis of early cervical cancer in stage I.

\begin{tabular}{llrr}
\hline Factors & \multicolumn{1}{c}{ MRI } & CT & $\chi^{2}$ value \\
\hline Sensitivity & $75.00 \%(54 / 64)$ & $62.50 \%(40 / 64)$ & 7.850 \\
Specificity & $72.92 \%(70 / 96)$ & $56.25 \%(54 / 96)$ & 0.005 \\
Diagnostic accordance rate & $77.50 \%(124 / 160)$ & $58.75 \%(94 / 160)$ & 0.016 \\
\hline
\end{tabular}

MRI, magnetic resonance imaging; CT, computed tomography.

Table VII. Diagnostic efficacy of MRI in lymphatic metastasis of early cervical cancer in stage II.

\begin{tabular}{lccr}
\hline & \multicolumn{2}{c}{ Pathological diagnostic results } & \multicolumn{2}{c}{ Stage non-IIa-IIb } & Total \\
\cline { 2 - 4 } Grouping & Stage IIa-IIb & 14 & 70 \\
\hline IIa-IIb phase by MRI diagnosis & 76 & 50 & 90 \\
Non-IIa-IIb phase by MRI diagnosis & 20 & 64 & 160 \\
Total & 96 & 0
\end{tabular}

MRI, magnetic resonance imaging.

Table VIII. Diagnostic efficacy of CT in lymphatic metastasis of early cervical cancer in stage II.

Pathological diagnostic results

Grouping

Stage IIa-IIb

Stage non-IIa-IIb

Total

IIa-IIb phase by CT diagnosis

60

36

20

88

Non-IIa-IIb phase by CT diagnosis

36
96

Total

64

160

CT, computed tomography. 
Table IX. Comparison of diagnostic efficiency of MRI and CT in lymphatic metastasis of early cervical cancer in stage II.

\begin{tabular}{|c|c|c|c|c|}
\hline Factors & MRI & CT & $\chi^{2}$ value & P-value \\
\hline Sensitivity & $79.17 \%(76 / 96)$ & $62.50 \%(60 / 96)$ & 6.454 & 0.011 \\
\hline Specificity & $78.13 \%(50 / 64)$ & $68.75 \%(44 / 64)$ & 1.442 & 0.230 \\
\hline Diagnostic accordance rate & $78.75 \%(126 / 160)$ & $58.75 \%(94 / 160)$ & 14.890 & $<0.001$ \\
\hline
\end{tabular}

MRI, magnetic resonance imaging; CT, computed tomography.

Table X. Diagnostic efficacy of MRI combined with CT in lymphatic metastasis of early cervical cancer in stage I.

\begin{tabular}{lccr}
\hline & \multicolumn{2}{c}{ Pathological diagnostic results } \\
\cline { 2 - 4 } Grouping & Stage Ia-Ib & Stage non-Ia-Ib & 12 \\
Ia-Ib phase by combined diagnosis & 50 & 84 & 62 \\
Non-Ia-Ib phase by combined diagnosis & 14 & 96 & 160 \\
Total & 64 & 96 \\
\hline
\end{tabular}

MRI, magnetic resonance imaging; CT, computed tomography.

Table XI. Diagnostic efficacy of MRI combined with CT in lymphatic metastasis of early cervical cancer in stage II.

Pathological diagnostic results

Grouping

Stage IIa-IIb

Stage non-IIa-IIb

Total

IIa-IIb phase by combined diagnosis

$\begin{array}{rr}90 & 11 \\ 6 & 53 \\ 96 & 64\end{array}$

Non-IIa-IIb phase by combined diagnosis

96

64

MRI, magnetic resonance imaging; CT, computed tomography.

Table XII. Comparison of the diagnostic efficacy between combined diagnosis with MRI or CT alone.

\begin{tabular}{|c|c|c|c|c|}
\hline Factors & Combination & MRI & CT & P-value \\
\hline \multicolumn{5}{|l|}{ Stage Ia-Ib } \\
\hline Sensitivity & $78.13 \%(50 / 64)$ & $75.00 \%(54 / 64)$ & $62.50 \%(40 / 64)$ & 0.013 \\
\hline Specificity & $87.50 \%(84 / 96)$ & $72.92 \%(70 / 96)$ & $56.25 \%(54 / 96)$ & $<0.001$ \\
\hline Diagnostic accordance rate & $83.75 \%(134 / 160)$ & $77.50 \%(124 / 160)$ & $58.75 \%(94 / 160)$ & $<0.001$ \\
\hline \multicolumn{5}{|l|}{ Stage IIa-IIb } \\
\hline Sensitivity & $91.66 \%(90 / 96)$ & $79.17 \%(76 / 96)$ & $62.50 \%(60 / 96)$ & $<0.001$ \\
\hline Specificity & $82.81 \%(53 / 64)$ & $78.13 \%(50 / 64)$ & $68.75 \%(44 / 64)$ & 0.161 \\
\hline Diagnostic accordance rate & $88.13 \%(143 / 160)$ & $78.75 \%(126 / 160)$ & $58.75 \%(94 / 160)$ & $<0.001$ \\
\hline
\end{tabular}

MRI, magnetic resonance imaging; CT, computed tomography.

improved when they are combined. Therefore, we consider that MRI and CT should be combined in clinic to improve the diagnostic accuracy of diseases.

\section{Acknowledgements}

Not applicable. 


\section{Funding}

No funding was received.

\section{Availability of data and materials}

The datasets used and/or analyzed during the current study are available from the corresponding author on reasonable request.

\section{Authors' contributions}

LL wrote the manuscript, interpreted and analyzed the data. QL designed the study and performed the experiments. LT was responsible for the analysis and discussion of the data. All authors read and approved the final manuscript.

\section{Ethics approval and consent to participate}

The study was approved by the Ethics Committee of Jining No. 1 People's Hospital (Jining, China). Patients who participated in this research had complete clinical data. Signed informed consents were obtained from the patients and/or the guardians.

\section{Patient consent for publication}

Not applicable.

\section{Competing interests}

The authors declare that they have no competing interests.

\section{References}

1. Small W Jr, Bacon MA, Bajaj A, Chuang LT, Fisher BJ, Harkenrider MM, Jhingran A, Kitchener HC, Mileshkin LR, Viswanathan AN, et al: Cervical cancer: A global health crisis Cancer 123: 2404-2412, 2017.

2. Wentzensen N, Schiffman M, Palmer T and Arbyn M: Triage of HPV positive women in cervical cancer screening. J Clin Virol 76 (Suppl 1): S49-S55, 2016.

3. Denny L: Cervical cancer: Prevention and treatment. Discov Med 14: 125-131, 2012.

4. Sreedevi A, Javed R and Dinesh A: Epidemiology of cervical cancer with special focus on India. Int J Womens Health 7 405-414, 2015.

5. Dornhöfer $\mathrm{N}$ and Höckel M: New developments in the surgical therapy of cervical carcinoma. Ann NY Acad Sci 1138: 233-252, 2008.

6. Tsili AC, Tsangou V,Koliopoulos G, Stefos Tand Argyropoulou MI: Early-stage cervical carcinoma: The role of multidetector CT in correlation with histopathological findings. J Obstet Gynaecol 33: $882-887,2013$

7. Hosaka M, Watari H, Mitamura T, Konno Y, Odagiri T, Kato T, Takeda M and Sakuragi N: Survival and prognosticators of node-positive cervical cancer patients treated with radical hysterectomy and systematic lymphadenectomy. Int J Clin Oncol 16: 33-38, 2011.

8. Petersen LJ, Nielsen JB, Langkilde NC, Petersen A, AfsharOromieh A, De Souza NM, De Paepe K, Fisker RV, Arp DT, Carl J, et al: ${ }^{68} \mathrm{Ga}$-PSMA PET/CT compared with MRI/CT and diffusion-weighted MRI for primary lymph node staging prior to definitive radiotherapy in prostate cancer: a prospective diagnostic test accuracy study. World J Urol, 2019. doi: 10.1007/ s00345-019-02846-z. [Epub ahead of print].
9. Kinahan PE, Townsend DW, Beyer T and Sashin D: Attenuation correction for a combined 3D PET/CT scanner. Med Phys 25: 2046-2053, 1998.

10. Shin S, Pak K, Kim SJ, Kim H and Kim SJ: Pulmonary tumor embolism derived from stomach cancer observation with serial ${ }^{18}$ F-FDG PET/CT. Clin Nucl Med 40: 270-272, 2015.

11. Nie D, Cao X, Gao Y, Wang L and Shen D: Estimating CT image from MRI data using 3D fully convolutional networks. Deep Learn Data Label Med Appl (2016) 2016: 170-178, 2016.

12. Low RN and Gurney J: Diffusion-weighted MRI (DWI) in the oncology patient: Value of breathhold DWI compared to unenhanced and gadolinium-enhanced MRI. J Magn Reson Imaging 25: 848-858, 2007.

13. Marth C,LandoniF,MahnerS,McCormack M,Gonzalez-Martin A and Colombo N; ESMO Guidelines Committee: Cervical cancer: ESMO Clinical Practice Guidelines for diagnosis, treatment and follow-up. Ann Oncol 28 (Suppl 4): iv72-iv83, 2017.

14. Landy R, Pesola F, Castañón A and Sasieni P: Impact of cervical screening on cervical cancer mortality: Estimation using stage-specific results from a nested case-control study. Br J Cancer 115: 1140-1146, 2016.

15. Lucia F, Visvikis D, Desseroit MC, Miranda O, Malhaire JP, Robin P, Pradier O, Hatt M and Schick U: Prediction of outcome using pretreatment ${ }^{18} \mathrm{~F}$-FDG $\mathrm{PET} / \mathrm{CT}$ and MRI radiomics in locally advanced cervical cancer treated with chemoradiotherapy. Eur J Nucl Med Mol Imaging 45: 768-786, 2018.

16. Choi J, Kim HJ, Jeong YH, Lee JH, Cho A, Yun M, Lee JD, Kim YB, Kim YT and Kang WJ: The role of ${ }^{18} \mathrm{~F}$-FDG PET/CT in assessing therapy response in cervix cancer after concurrent chemoradiation therapy. Nucl Med Mol Imaging 48: 130-136, 2014.

17. Patel CN, Nazir SA, Khan Z, Gleeson FV and Bradley KM: ${ }^{18} \mathrm{~F}-\mathrm{FDG} \mathrm{PET} / \mathrm{CT}$ of cervical carcinoma. AJR Am J Roentgenol 196: 1225-1233, 2011.

18. Kim HS, Lee KS, Ohno Y, van Beek EJ and Biederer J: PET/CT versus MRI for diagnosis, staging, and follow-up of lung cancer. J Magn Reson Imaging 42: 247-260, 2015.

19. Shen G, Deng H, Hu S and Jia Z: Comparison of choline-PET/CT, MRI, SPECT, and bone scintigraphy in the diagnosis of bone metastases in patients with prostate cancer: A meta-analysis. Skeletal Radiol 43: 1503-1513, 2014.

20. Sawicki LM, Grueneisen J, Schaarschmidt BM, Buchbender C, Nagarajah J, Umutlu L, Antoch G and Kinner S: Evaluation of ${ }^{18}$ F-FDG PET/MRI, ${ }^{18}$ F-FDG PET/CT, MRI, and CT in whole-body staging of recurrent breast cancer. Eur J Radiol 85: 459-465, 2016.

21. Cazzato RL, Garnon J, Shaygi B, Koch G, Tsoumakidou G, Caudrelier J, Addeo P, Bachellier P, Namer IJ and Gangi A: PET/CT-guided interventions: Indications, advantages, disadvantages and the state of the art. Minim Invasive Ther Allied Technol 27: 27-32, 2018

22. Yong HE: Comparative analysis of diagnostic value of imaging of cervical cancers by B-ultrasonography, CT and MRI. Acta Med Sin 03: 413-415, 2009

23. Li LC and Room C: Clinical value of preoperative CT and MRI on diagnosing invasive cervical cancer. Han Shao Ji Bing Za Zhi 01: 62-63, 2018 (In Chinese).

24. Heacock L, Weissbrot J, Raad R, Campbell N, Friedman KP, Ponzo F and Chandarana H: PET/MRI for the evaluation of patients with lymphoma: Initial observations. AJR Am J Roentgenol 204: 842-848, 2015.

25. Bin D and Li ZL: Value analysis and efficacy observation of CT in combination with MRI on staging diagnosis of endometrial carcinoma. Chin J Ct Mri 04: 107-109, 2016.

26. Kim YN, Yi CA, Lee KS, Kwon OJ, Lee HY, Kim BT, Choi JY, Kim SW, Chung MP, Han J, et al: A proposal for combined MRI and PET/CT interpretation criteria for preoperative nodal staging in non-small-cell lung cancer. Eur Radiol 22: 1537-1546, 2012.

27. Ren F, Gao L, Yao FM, Hu TB and Lin MJ: The technological efficacy of CT combined with MRI in diagnosing calcified meningioma. Health Res 3: 259-260, 2014.

This work is licensed under a Creative Commons Attribution-NonCommercial-NoDerivatives 4.0 International (CC BY-NC-ND 4.0) License. 